\title{
Determination of the pipe stemming load
}

\author{
S. C. Cowin
}

\section{MASTER}

\section{October 19, 1979}




\title{
Determination of the pipe stemming load
}

\author{
S. C. Cowin*
}

Mamuscript date: October 19, 1979

-Present Adiress: Tulnne University, New Orleans, LA

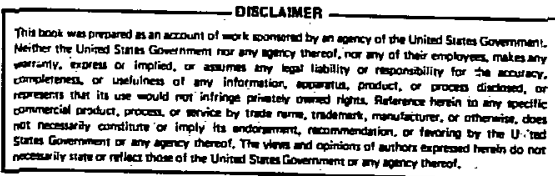

LAWRENCE LIVERMORE LABORATORY University of California - Livermore, California • 94550 


\section{CONTENTS}

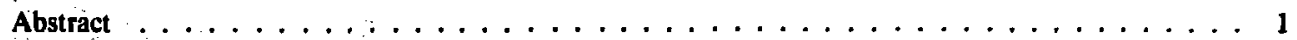

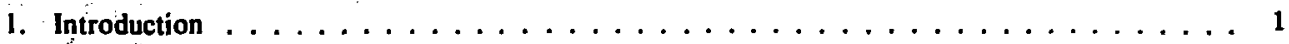

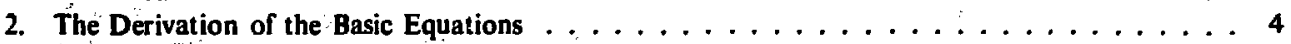

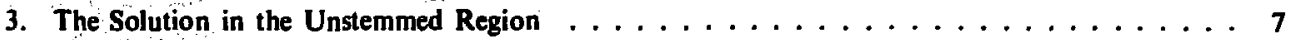

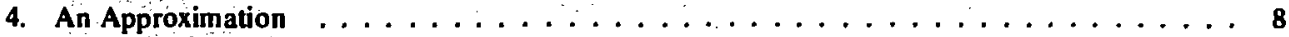

5. The Solution in the Lower Portion of the Stemmed Region When Slipping Occurs on the Pipe-Stemming Interface . . . . . . . . . . . . 9

6. The Solution in the Upper Portion of the Stemmed Region Where Slip Does Not Occur on the Pipe-Stemming Interface ............. 10

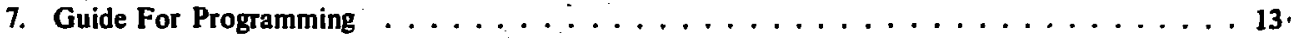

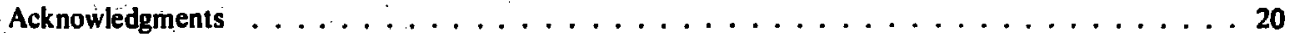

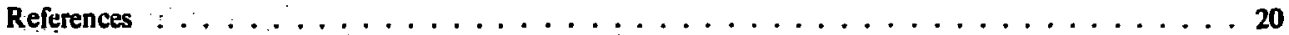

Appendix I-The Solution without the Approximation . . . . . . . . . . . . 21

1-1. The Solution in the Lower Portion of the Stemmed Region

When Slipping Occurs on the Pipe-Stemming Interface $\ldots \ldots \ldots \ldots$

I-2. The Solution in the Upper Portion of the Stemmed Region

Where Slip Does Not Occur on the Pipe-Stemming Interface $\ldots \ldots \ldots$

Appendix II-The Effect of Temperature on the Determination of the

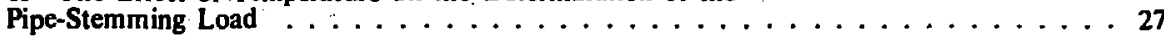




\section{DETERMINATION OF THE PIPE STEMMING LOAD}

\section{ABSTRACT}

A mechanical model for the emplacement pipe system is developed. The model is then employed to determine the force applied to the surface collar of the emplacement pipe, the pipe-stemming load, and the stress along the emplacement pipe as a function of stemming height. These results are presented as integrals and a method for their numerical integration is given.

\section{INTRODUCTION}

The pipe-stemming load is the portion of the weight of the stemming material carried by the emplacement pipe in a vertical underground test system. The emplacement-pipe supports the experimental device from above. The hole containing the emplacement pipe and experimental device is backfilled with stemming material to prevent the escape of material during and after the test. Hamilton ${ }^{1}$ did the initial analytical work to determine the pipe stemming load. He suggested that the stemming load, i.e., the weight of the stemming material used to backfill the emplacement hole, was partially carried by the pipe support system and partially carried by the wall of the emplacement hole. The portion of a total increment in stemming weight carried by the pipe support system was argued by Hamilton to be $\mathbf{R}_{p} /\left(\mathbf{R}_{\mathbf{p}}+\mathbf{R}_{h}\right)$, where $\mathbf{R}_{\mathbf{p}}$ and $\mathbf{R}_{h}$ are the pipe and hole radii, respectively. The ratio $\mathbf{R}_{p} /\left(\mathbf{R}_{\mathrm{p}}+\mathbf{R}_{h}\right)$ is called the werted perimeter fraction. The stemming tests of $\mathrm{Cramer}^{2}$ on a 100 -ft-length of pipe apparently confirmed the wetted perimeter concept. However, Norris ${ }^{3}$ observed that the collar stemming load data from a number of emplacement systems contradicted Cramer's results. The increase in the collar stemming load in the actual emplacement system was considerably less than that predicted by the wetted perimeter concept. Norris surmised that the discrepancy between the actual emplacement pipe collar data on one hand and Hamilton's wetted perimeter concept and Cramer's experimental data on the other hand was caused by Cramer and Hamilton not accounting for the "load release due to pipe movement." That is, the downward motion of the support pipe through the stemming material transferred some of the total load carried by the pipe to the emplacement hole wall through the stemming material. Norris observed that the short pipe used by Cramer had an extremely high stiffness compared to the usual systems of long emplacement pipes. Norris states that a prime mechanism for reducing the collar stemming load appears to be fisplacement of the pipe. A second mechanism is the support given by stemming material under and around the sides of the device-diagniostic package.

Norris presented a more refined model for the pipe-stemming load: He assumed that the pipe stemming load was reduced by "a concentrated spring at the (device-diagnostic) canister with reactive force proportional to the displacement associated with the concentrated canister stemming load" He assumed that the weight of the stemming material carried by the pipe was reduced by the concentrated canister spring and by a distributed spring,called $\mathbf{K}_{\text {soil }}$ in the annulus stemming. $\mathbf{K}_{\text {soil }}$ provided a restoring force proportional to the displacement associated with the wetted perimeter-stemming load. Norris' work led to the development of Stem Code, a computer program to determine the pipe stemming load: An early version of this program is described in Ref. 4.

At that time Stem Code and its rationale was a major advance on the pioneering work by Hamilton. However, the predictions of Stem Code for the pipe stemming load did not agree with the measured data. Modifications of the code were made and new parameters were introduced Blakes reviewed the situation in 1973. He observed that the spring constant $\mathbf{K}_{\text {soil }}$ introduced in the Steri Code could be adjusted to make the 
predictions of the Stem Code agree with the experimental data. In some cases there was still no agreement between Stem Code and the measured pipe collar loads; the Portulaca event was cited as an example. Blake also noted that the Stem Code predictions were relatively insensitive to many code parameters, and the parameter $\mathbf{K}_{\text {soil }}$ had no basis in experimental fact.

The present work resulted from a meeting on July 13, 1977 of A. Blake, R. Corallo, D. Maiden, F. Morrison and S. C. Cowin. Two changes in the analysis for the pipe-stemming load calculation were discussed. The first was removal of the assumption that the vertical stress in the stemming was constant, and the second was adjustment of the zero-strain reference staine in the stemming material to be consistent with the motion of the support pipe. On July 15,1977 , Cowin wrote a note to the participants in which the goveming differential equations were derived, but not solved. It was implied that, since the governing differential equations were linear, the problem might be solved easily. However, the solution of the problem was not simple, as this repoit shows.

This analysis is an extension of those done by Hamilton ${ }^{1}$ and Norris. ${ }^{3}$ The differences lie in the details of the assumptions. In particular, the idea of Norris ${ }^{3}$ concerning load release caused by pipe movement is pursued to better understanding of the interaction between the emplacement pipe and the stemming material. Our analysis suggests that load release caused by pipe movement is in excess of that suggested by Norris. The mechanism for this load release is the reversal of the direction of the friction force between the stemming material and the pipe. This occurs because the low stiffness of the long pipe permits large relative deflections of the distal end of the pipe. The contrast in the situation experienced by the near and far end of a highly stemmed long pipe is illustrated in Fig. 1 . To demonstrate that this occurs in practice, note that the deflection $\delta$ of the steel pipe $1,500 \mathrm{ft}$ long with a cross-sectional area of $16 \mathrm{in}^{2}$ is $3.5 \mathrm{in}$. caused by $2 / 3$ of its wetted perimeter load.

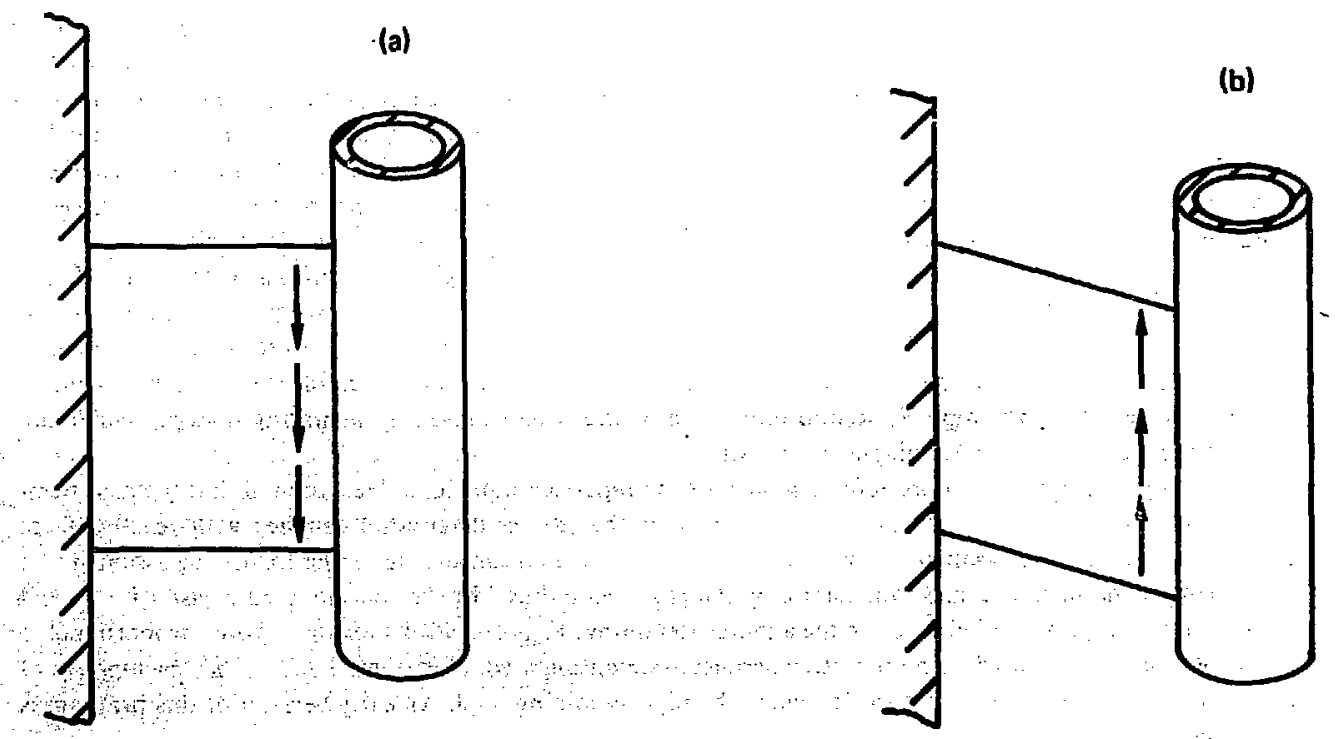

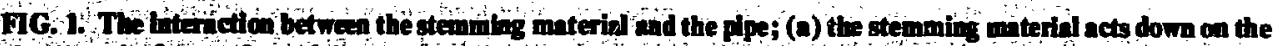

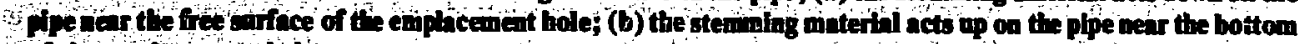

tof the plocepent bole. 


$$
\delta=\frac{P L}{A E}=\frac{(94,000 \mathrm{lb})(1500 \mathrm{ft} \times 12 \mathrm{in} / \mathrm{ft})}{\left(16 \mathrm{in.} .^{2}\right)(30,000,000 \mathrm{psi})}=3.5 \mathrm{in} .
$$

where

$$
P=\frac{R_{p}}{R_{h}-R_{p}}\left(R_{h}^{2}-R_{p}^{2}\right) \gamma \times 1000 \mathrm{ft}=94,000 \mathrm{lb}
$$

On the other hand, the maximum deflection of the stemming material before slip occurs between the pipe and the stemming material is $\sim 1$ in. Actually, it is considerably less than 1 in and the greatest leeway is taken to raise the deflection to 1 in. Thus, it is reasonable to expect that for a long pipe stemmed for a large portion of its length, the effective direction of the pipe-stemming material friction force can reverse itself.

The consequences of this reversal of friction force are significant. For example, the portion of a total increment in stemming weight carried by the pipe support system need not be in the ratio $\mathbf{R}_{\mathrm{p}} /\left(\mathbf{R}_{\mathrm{p}}+\mathbf{R}_{\mathrm{h}}\right)$, but can te variable and can be a negative number. The wetted perimeter concept holds only for short pipes, not for the long pipes of an emplacement system. It is reasonable to expect that the pipe collar load could vanish. Repor ts have indicated that this has been observed. For example, strong backs supporting the load have, on some events, been removed after the stemming operation was complete.

The interaction mechanism between the stemming material and the support pipe is treated in detail in this work. Norris ${ }^{3}$ realized that the downward nsfiection of the pipe induced a shearing strain in the surrounding stemming material annulus. The zero-strain reference state for the stemming material was taken as the unstenmed pipe deflection at the point in question. Here the zero-strain reference state in the stemming material at a given downhole location is the pipe deflection when the support pipe has been stemmed to that particular downhole location.

The modeled interfacial interaction between the stemming material and the pipe is now described. It is assumed that when the stemming material is emplaced at a specified location, the stemming material exerts a downward force on the pipe. This downward force is assumed to be the maximum that can occur without slipping. As the stemming level rises above this location, the pipe deflects in the direction of the downward force exerted by the stemming material. As the stemming level rises farther, the pipe continues to deflect. The force on the pipe caused by the stemming material decreases and vanishes as the strained stemming material takes the weight of the stemming material previously carried by the pipe. As stemming continues above the specified location, the pipe deflection increases and the force of the stemming material on the pipe reverses its direction and acts upward on the support pipe, opposing its motion. The final stage of the interfacial interaction between the pipe and stemming material occurs when the static friction force is overcome and the pipe slides down theough the stationary stemming. The considerations of these mechanisms set this work apart from its predecessors.

The principal results of this report are now described. The input parameters that determine the results and are assumed to be known are the following.

$E=$ Young's modulus for the pipe.

$A=$ Cross-sectional area of the pipe.

$w=$ Weight per unit length of the pipe.

$\mathbf{R}_{\mathrm{p}}=$ Radius of the pipe.

$\mathbf{R}_{\mathrm{h}}=$ Radius of the emplacement hole.

$\mu_{p}=$ Coefficient of friction between the stemming and the pipe.

$\mu_{h}=$ Coefficient of friction between the stemming and the hole wall.

$\mathbf{K}=$ Ratio of lateral to vertical pressure in the stemming material (Janssen's coefficient).

$\mathbf{G}=$ Shear modulus of the stemming material.

$L=$ Length of the pipe. 
$W=$ Weight of the device-diagnostic canister and other load applied at the end of the pipe.

$u_{R}(s)=H$ istory of release displacement at the pipe collar given as a function of $\mathrm{s}$, the downhole distance to the free surface of the stemming material.

The solution is determined by two functions $y(s)$ and $F(s)$, where $s$ is the downhole distance to the free surface of the stemming. The distance from the free surface of the stemming to the point where the pipe begins to slip relative to the stemming material is $y . F$ is the force at the pipe support collar and the focus of this analysis. To determine $F(s)$, y(s) must be deter mined first. $F(s)$ is given by $E q$. (46) and $y(s)$ by Eq. (50). The solution of these equations is not difficult with the present computational facilities.

The derivation of the basic equations is summarized in Sec. 2. These equations are solved in the unstemmed region in Sec. 3, and an approximation to the equations is given in Sec. 4. The solution in the lower portion of the stemmed region where slipping occurs on the pipe-stemming interface is presented in Sec. 5 . In Sec. 6 the solution in the upper portion of the stemmed region where slip does not occur on the pipe-stemming interface is developed. Appendix I presents the solution in the case where the approximation of Sec. 4 is not introduced and Appendix II deals with the inclusion of thermal effects.

\section{THE DERIVATION OF THE BASIC EQUATIONS}

The problem is divided into two parts for this analysis. Consider the partially stemmed hole shown in Fig. 2. The portion of the pipe that is unstemmed lies between 0 and the level of the free surface of the stemming material $s, 0<x<s$. The stemmed portion of the pipe is between $s$ and $L$, i.e, all stations $x$ such that $s<\mathrm{x}<\mathrm{L}$. The vertical displacement of the pipe at the station $\mathrm{x}$ due to stemming up to the station $s$ is denoted

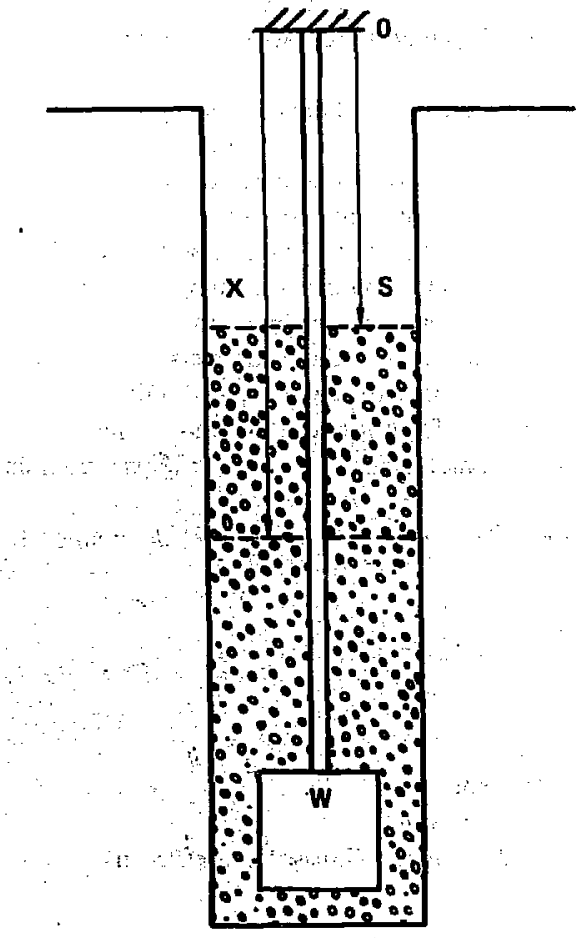

FIG. 2. A schematic of a partially stemmed hole. 
by $u(x, s)$. Similarly, we let $T(x, s)$ denote the axial force in the pipe at a station $x$ due to stemming up to the station $s$. If the hole is unstemmed, then the displacement is denoted by $u(x, L)$ and the axial force by $T(x, L)$. The axial force $T(x, s)$ and the vertical displacement are related by Hooke's law

$$
T(x, s)=E A \frac{d u}{d x}(x, s)
$$

where $\mathbf{E}$ denotes Young's modulus for the pipe and $A$ is the cross-sectional area of the pipe.

Equilibrium of the pipe requires that $T(x, s)$ satisfy the equation

$$
\frac{d T(x, s)}{d x}+w=0, \text { if } 0 \leqslant x \leqslant s
$$

or

$$
\frac{d T(x, s)}{d x}+2 \pi R_{p} f(x, s)+w=0, \text { if } s \leqslant x \leqslant L,
$$

where $t(x, s)$ is the shear stress exerted on the pipe by the stemming material, $w$ is the weight per unit length of the pipe, and $R_{p}$ is the radius of the pipe. The derivation of Eq. (2b) from a free-body diagram of a pipe section is shown in Fig. 3. Equation (2a) follows from Eq. (2b) when $\tau(x, s)$ vanishes. A similar free-body diagram of an annular-shaped slice of stemming material in Fig. 4 shows that

$$
\frac{d \sigma}{d x}(x, s)+\frac{\sigma(x, s)}{l_{1}}+\frac{2 R_{p}}{R_{h}^{2}-R_{p}^{2}} \tau(x, s)-\gamma=0, \text { if } s \leqslant x \leqslant L
$$

and

$$
\sigma(x, s)=0, \text { if } 0 \leqslant x \leqslant s
$$

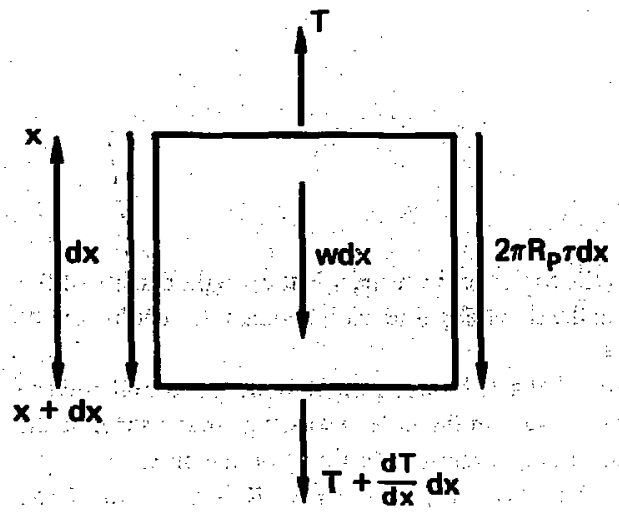

FIG. 3. A free-body agram of the pipe. The saim of the forces for this free-body dingran is:

$T+d T / d x d x-T+2 \pi R_{p}$ rdx $+w d x=0$

or

$\mathrm{d} / \mathrm{dx}+2 \pi \mathrm{R}+\mathrm{T}=0$ Where $P$ is the $a x i f$ force in the pipe, $R \&$ be ralles of the plpe, $w$ to the thefht per unit le gft of the plpe, and $r$ is the shear stress the stenmitg mitheill cleces on the plpe. 


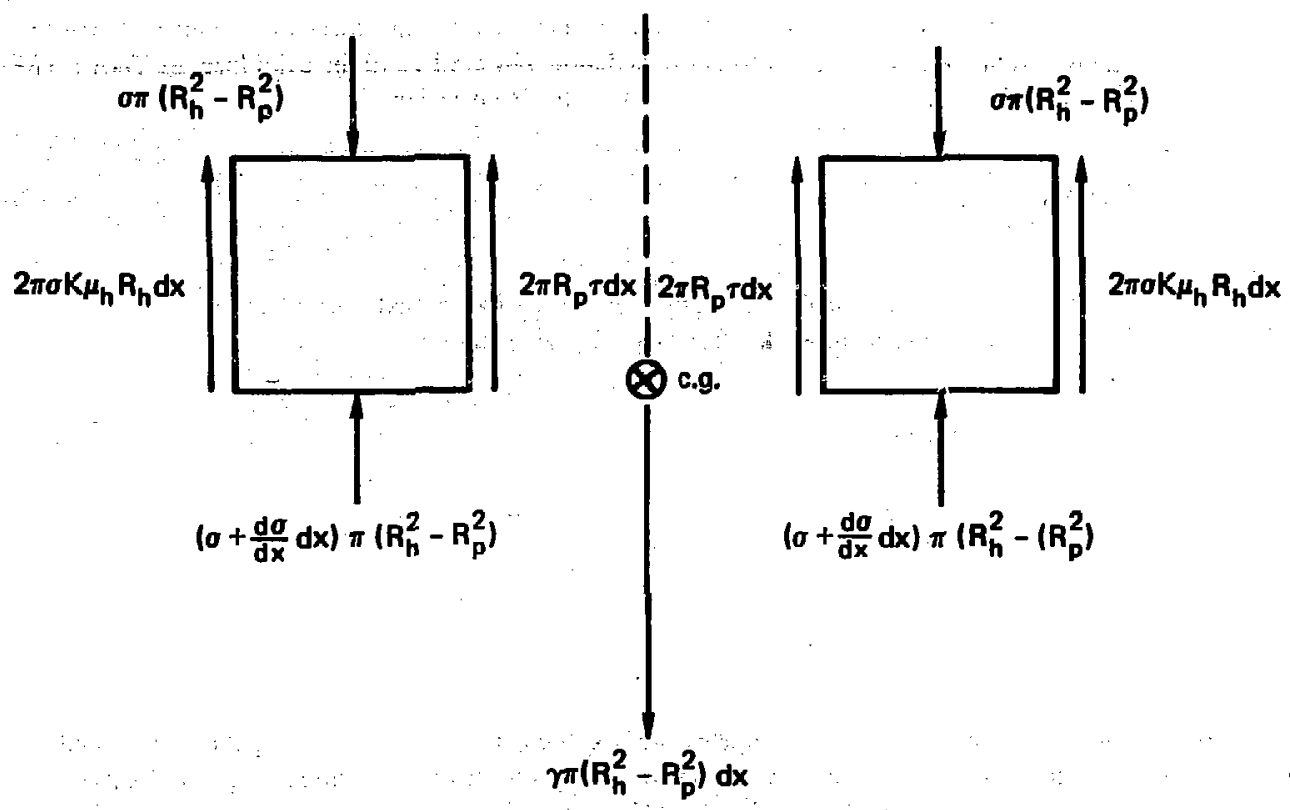

FIG. 4. A free-hody diagram of an amolar-shaped volume of stemming materinl. The sam of the forces for this free-body dogram is:

$$
\begin{aligned}
&\left(\sigma+\frac{d \sigma}{d x} d x\right) \pi\left(R_{h}^{2}-R_{p}^{2}\right)-\sigma \pi\left(R_{h}^{2}-R_{p}^{2}\right)+2 \pi R_{p} \pi d x+\mu_{h} R_{h} 2 \pi K \sigma d x \\
&-\gamma \pi\left(R_{h}^{2}-R_{p}^{2}\right) d x=0, \text { or } \frac{d \sigma}{d x}+\frac{2 \mu_{h} R_{h} K}{R_{h}^{2}-k_{p}^{2}} \sigma+\frac{2 R_{p}}{R_{h}^{2}-R_{p}^{2}} \tau-\gamma=0,
\end{aligned}
$$

where $\sigma$ is the vertical compressive stress in the stemming, $\gamma$ is the weight density of the stemming material, $\mathbf{R}_{\mathrm{h}}$ is the ralius of the hoie, $\mathbf{K} \mathrm{b}$ the ratis sf horizonthl pressure to vertical preseure in the stemning, and $\mu_{\mathrm{h}}$ is the coefficient of friction between the stemining and the casing wall.

where

$$
\ell_{1}=\frac{R_{h}^{2}-R_{p}^{2}}{2 \mu_{h} R_{h}^{K}}
$$

and where of $x, s)$ is the vertical striss in the stemming, $R_{h}$ is the radius of the hole, $\gamma$ is the weight density of the stemming, $\mathbf{K}$ is the ratio of horis tallo vertical pressure in the stemming material, and $\mu_{h}$ is the coefficient of friction between the stemming nuaterial and the hole wall.

The assumption about the nature of the shear stress that acts between the stemming material and the pipe is now given in detail. As the stemming material falls into place in the hole, we assume that a shear stress tof maximum magnitude develops and it acts up on the stemming material and down on the pipe, i.e., in the direction of positive $\tau$. The maximum magnitude of the shear stress is $\mu_{\mathrm{p}} \mathrm{K}_{\sigma}$, where $K$ is the ratio of the 
horizontal to vertical pressure and $\mu_{\mathrm{p}}$ is the coefficient of friction between the pipe and the stemming material. These assumptions are the same as those used to obtain Janssen's formulas. ${ }^{6-8}$. The situation changes as the hole fils, because the deflection of the pipe at previously filled stations is increasing and the pipe tends to move down relative to the stemming material, reducing the shear stress $\tau$. As the downward deflection of the pipe is increased, the shear stress $\tau$ changes sign and then increases to a maximum negative magnitude $-\mu_{p} K \sigma$. Further movement of the pipe occurs by slipping through the stemming material. It is assumed that the shear stress $\tau$ changes from $+\mu_{\mathrm{p}} \mathrm{K} \sigma$ to $-\mu_{\mathrm{p}} \mathrm{K} \sigma$ smoothly and linearly because of the elasticity of the stemming material in shear. With these assumptions the shear stress $\tau$ is given by

$$
\begin{aligned}
& \tau(x, s)=\mu_{p} K \sigma-\frac{G}{R_{h}-R_{p}}[u(x, s)-u(x, x)], \\
& \text { if } \tau \geqslant-\mu_{p} K \sigma \text { and } s \leqslant x \leqslant L ;
\end{aligned}
$$

otherwise there is a slip displacement $u_{\text {slip }}$ at the pipe surface given by

$$
u_{\text {sip }}=u(x, s)-u(x, x)-\frac{\left(R_{h}-R_{p}\right)}{G} 2 \mu_{p} K \sigma(x, s), \text { if } s \leqslant x \leqslant L .
$$

In Eqs. (5) and (6), $G$ is the shear modulus of the stemming material and $u(x, x)$ is the deflection of the pipe at a station $x$ when the free surface of stemming is at station $x$. The displacement $u(x, x)$ is subtracted from $u(x, 3)$ to obtain a zero-strain initial reference strain state for the stemming material.

The system of equations describing the behavior of the pipe and stemming material are Eqs. (I), (2b), (3), (5), and (6). These equations apply to the stemmed portion of the pipe. Equations (1) and (2a) apply to the unstemmed portion.

\section{THE SOLUTION IN THE UNSTEMMED REGION}

The vertical displacement $u(x, s)$ and the axial pipe force $T(x, s)$ in the unsterimed region of the pipe can be determined from Eqs. (1) and (2a), subject to two boundary conditions. We let $F(s)$ denote the axial force at the top of the pipe and $u_{R}$ denote the release displacement, i.e., the adjusted displacement of the pipe at the top of the hole obtained by releasing the pipe after the stemming has been initiated,

$$
\underbrace{u(0, s)=u_{R}(s),}_{1} \underbrace{T(0, s)=F(s)}_{2}
$$

Equation (2a) can be integrated directly using the boundary condition Eq. (7),

$$
T(x, s)=F(s)-w x, \quad 0 \leqslant x \leqslant s .
$$

A differential equation is cbtained from Eqs. (1) and (2a) by the elimination of $T(x, s)$,

$$
E A u^{\prime \prime}(x ; s)+w=0
$$

This differential equation may be integrated using the boundary conditions in Eq. (7) and using Eq. (2a),

$$
u(x, s)=\frac{F(s)}{E A} x-\frac{w}{2 E A} x^{2}+u_{R}(s), \text { if } 0 \leqslant x<s
$$


If the entire length of the pipe is unstemmed, then the tension $T(L, L)$ in the pipe at the bottom must equal the weight of the device diagnostic package W; thus from $\mathrm{Eq}_{\text {( }}(8)$

$$
\mathbf{T}(\mathbf{L}, \mathbf{L})=\mathbf{W}=\mathbf{F}(\mathbf{L})-\mathbf{w} \mathbf{L}
$$

The deflection $u(x, L)$ in the case when the entire length of pipe is unstemmed is then given by the following expression

$$
u(x, L)=\frac{W x}{A E}+\frac{W x}{A E}\left(L-\frac{x}{2}\right)+u_{R}(L)
$$

where $F(L)$ from Eq. (11) has been substituted into Eq. (10) evaluated at $s=\mathrm{L}$. It is reasonable to assume that $u_{R}(L)=0$ and that will be done in a later section.

\section{AN APPROXIMATION}

The solution to the complete system of equations is complicated, but not impossible. A solution is described in Appendix $I$. In this section it is shown that a reasonable approximation in the basic system of equations will reduce the complexily of the calculations. This approximation is based on the observation that the first tern in Eq. (3) is small compared to the other three terms, thus Eq. (3) can be approximated by

$$
\frac{\sigma(x, s)}{l_{1}}+\frac{2 R_{p}}{R_{h}^{2}-R_{p}^{2}} \tau(x, s)-\gamma=0, \text { if } s \leqslant x \leqslant L
$$

A dimensional analysis of Eq. (3) shows that the first term is proportional to $\ell_{1} / \ell_{0}$, wher $\ell_{0}$ is a length used to render the $x$ coordinate dimensionless. For a typical stemming operation $R_{h}=36$ in., $R_{p}=5$ in., and $\mu_{h} K \cong$ 1/4; thus from Eq. (4) $l_{1}$ is about $6 \mathrm{ft}$. It is reasonable to select $l_{0}$ much greater than $6 \mathrm{ft}$, thus $l_{1} / l_{0}$ is small and the derivative of $\sigma(x, s)$ with respect to $x$ can be neglected. This does not mean that $\sigma(x, s)$ is a constant; only that its first derivative is smaller than the other terms of Eq. (3) in the problem under consideration.

Equations (13) and (5) may be solved for $\tau(x, s)$ and $\sigma(x, s)$ in terms of dispiacement $u(x, s)$,

$$
\tau(x, s)=\frac{\gamma \mu_{p} K\left(R_{h}^{2}-R_{p}^{2}\right) R_{1}}{R_{h}^{2}-R_{p}^{2}+2 R_{p} \mu_{p} K l_{1}}-\frac{C\left(R_{h}+R_{p}\right)}{R_{h}^{2}-R_{p}^{2}+2 R_{p} \ell_{1}}[u(x, s)-u(x, x)],
$$

and

$$
\sigma(x, s)=\frac{\left(R_{h}^{2}-R_{p}^{2}\right) \gamma_{1}}{R_{h}^{2}-R_{p}^{2}+2 R_{p} \mu_{p} K \ell_{1}}+\frac{2 R_{p} \ell_{l} G}{\left(R_{h}-R_{p}\right)\left(R_{h}^{2}-R_{p}^{2}+2 R_{p} \ell_{1}\right)}[u(x, s)-u(x, x)]
$$




\section{THE SOLUTION IN THE LOWER PORTION OF THE STEMMED REGION WHEN SLIPPING OCCURS ON THE PIPE-STEMMING INTERFACE}

The determiniation of the pipe displacement $r(x, s)$, the axial pipe force $T(x, s)$ and the vertical stemming material stress of $(x, s)$ is much more complicated in the stemmed region than is the determination of $u(x, s)$ and $T(x, s)$ in the unstemmed region. The problem is considered in two parts. First we consider the case when $\tau(x, s)=-\mu_{p} K \sigma(x, s)$ in the lower portion of the stemmed region and $\tau(x, s)>-\mu_{p} K \sigma(x, s)$ in the upper portion of the stemmed region. In this section the problem of slipping along the lower portion of the pipe stemming interface is considered. In the next section this result is used to obtain a solution to the problem in the no-slipping region above the slipping region.

Let $s+y, y>0$ denote the downhole distance at which slip first occurs, $0<s+y<x<L$. Slip is assumed to occur along the pipe for $x$ greater than $s+y$ and less than $L, s+y<x<L$. Since $r(x, s)=$ $-\mu_{p} K \sigma(x, s)$; it follows from Eq. (13) that

$$
\sigma(x, s)=\sigma_{0}, \tau(x, s)=-\mu_{p} K \sigma_{0},
$$

where

$$
\sigma_{0} \equiv \frac{\gamma_{1} \ell_{2}}{\ell_{2}-\ell_{1}}, \quad \ell_{2}=\frac{R_{h}^{2}-R_{p}^{2}}{2 \mu_{p} R_{p} K} .
$$

The slip displacement is given by Eq. (6),

$$
u_{d \mathrm{sip}}=u(x, s)-u(x, x)-\frac{R_{h}-R_{p}}{G} 2 \mu_{p} K_{o} .
$$

The equilibrium condition Eq. (2b) can $1 ;$ rewritten in the form

$$
\frac{d T(x, s)}{d x}+w_{e}=0,
$$

where

$$
w_{e}=w-2 \pi R_{p} \mu_{p} K q_{p}
$$

is the effective weight per unit length of the pipe. $w_{e}$ is less than $w$ in the region $s+y<x<L$ because the friction force is acting upward in this region, reducing th effective weight. The solution to Eq. (19) subject to the boundary condition

$$
\begin{gathered}
T(x, s) \mid=W \\
x=L
\end{gathered}
$$

is:

$$
T(x, s)=W+w_{e}(L-x) \text { for } s+y \leqslant x \leqslant L .
$$

Note that the pipe tension in the stemmed region of the pipe that has slipped is less than the tension in the same region with no slipping. 


\section{THE SOLUTION IN THE UPPER PORTION OF THE STEMMED REGION WHERE SLIP DOES NOT OCCUR ON THE PIPE-STEMMING INTERFACE}

The syatem of differential Eqs. (1), (2b), (5) and (13) can be reduced to the solution of a single second-order differential equation with constant coefficients for $u(x, s)$. To obtain this differential equation, first substitute Eqs. (1) and (5) into Eq. (2b) and solve for $\sigma\left(x_{j} s\right)$,

$$
\sigma(x, s)=\frac{E A l_{2}}{A l_{3}^{2}}[u(x, s)-u(x, x)]-\frac{l_{2}}{A_{s}}\left[E A u^{\prime \prime}(x, s)+w\right]
$$

where the following notations have been introduced:

$$
l_{3}=+\left(\frac{E A\left(R_{h}-R_{p}\right)}{2 \pi R_{p} G}\right)^{1 / 2}, A_{s}=\pi\left(R_{h}^{2}-R_{p}^{2}\right)
$$

The differential equation for $u(x, s)$ is oblained by substituting $\sigma(x, s)$ given by Eq. (23) and $\tau(x, s)$ given by Eq. (5) into Eq. (13),

$$
u^{\prime \prime}(x, s)-\lambda^{2} u(x, s)=-\frac{w}{E A}-\frac{\gamma A_{s}}{E A} \frac{l_{1}}{l_{1}+l_{2}}-\lambda^{2} u(x, x),
$$

where

$$
\lambda^{2}=\frac{l_{2}}{\left(l_{1}+l_{2}\right) R_{3}^{2}}
$$

and $\lambda$ has the dimension of reciprocal length. If we take a typical emplacement, assuming

$$
R_{h}=36 \text { in., } R_{p}=5 \text { in., } A=16 \text { in., } K \mu_{p} \cong K \mu_{n} \cong \frac{1}{4}, G=2000 \mathrm{psi} \text {, and } E=30 \times 10^{6} \text { psi, }
$$

then from Eqs. (4) and (24)

$$
l_{1}=5.88 \mathrm{ft}, \ell_{2}=42.36 \mathrm{ft} ; \mathrm{l}_{3}=40.55 \mathrm{ft} \text { and } \lambda= \pm(43.3 \mathrm{ft})^{-1}
$$

The value of $u(x, x)$ occurring in Eq. (25) can be obtained from Eq. (10), thus Eq. (25) can be rewritten as

$$
u^{\prime \prime}(x, s)-\lambda^{2} u(x, s)=-\frac{1}{E A}\left[w+\gamma A_{s} \frac{l_{1}}{l_{1}+l_{2}}+\lambda^{2} x F(x)-\lambda^{2} \frac{w x^{2}}{2}+\operatorname{EA\lambda }^{2} u_{R}(x)\right]
$$

This differential equation must satisfy the boundary conditions

$$
u(s, s)=\frac{s F(s)}{E A}-\frac{w s^{2}}{2 E A}+u_{R}(s)
$$

and

$$
u^{\prime}(s, s)=\frac{F(s)}{E A}-\frac{w}{E A} s,
$$


which are the values of $u(x, s)$ and $u^{\prime}(x, s)$ given by Eq. (10) evaluated at $x=s$. These conditions are consequences of the continuity of displacement and force at the interface $x-s$.

The solution to Eq. (29), subject to the boundary conditions Eq. (30), is

$$
\begin{aligned}
\operatorname{EAu}(x, s)= & \operatorname{EAu}_{R}(s) \cosh \lambda(x-s)+\frac{l_{3}^{2} l_{2}}{l_{2}} \gamma A_{s}[1-\cosh \lambda(x-s)] \\
& -\frac{w x^{2}}{2}+F(s)\left[\frac{1}{\lambda} \sinh \lambda(x-s)+s \cosh \lambda(x-s)\right] \\
& -\int_{s}^{x}\left[\xi F(\xi)+E A u_{R}(\xi)\right] \lambda \sinh \lambda(x-\xi) d \xi .
\end{aligned}
$$

This result is difficult to obtain and is checked by verifying that Eq. (31) satisfies Eq. (29) and the boundary conditions $\mathrm{Eq} .(30)$. It is helpful to keep in mind the following formula for the derivative of an integral. If

$$
G(x)=\int_{p(x)}^{q(x)} f(x, \xi) d \xi
$$

then

$$
G^{\prime}(x)=\int_{p(x)}^{q(x)} \frac{d f(x ; \xi)}{d x} d \xi+f[q(x), x] q^{\prime}(x)-f[p(x), x] p^{\prime}(x)
$$

A derivation of this result is given in Ref. 9. The term $\lambda \sinh \lambda(x-5)$ is the Green's iunction for the differential equation labeled as, Eq. (29).

The axial tension $T(x, s)$ in the pipc can be obtained by substituting Eq. (31) into Eq. (1)

$$
\begin{aligned}
& T(x, s)=\lambda \operatorname{EAu}_{R}(s) \sinh \lambda(x-s)-\frac{g_{3}^{2} \ell_{1}}{\ell_{2}} \gamma A_{s} \lambda \sinh \lambda(x-s)-w x+F(s)[\cosh \lambda(x-s) \\
&+\lambda s \sinh \lambda(x-s)]-\int_{s}^{x}\left[\xi F(\xi)+E A u_{R}(\xi)\right] \lambda^{2} \cosh \lambda(x-\xi) d \xi \text { for } s \leqslant x<s+y
\end{aligned}
$$

The condition to determine the function $F(s)$ is that $T(x, s)$, given by Eq. (34) and evaluated at $x=s+y$, must equal $T(x, s)$ given by - Eq. (22) and àlso evaluated at $x=s+y$

$$
\begin{aligned}
& w+w_{e}(L-s-y)=\lambda E A u_{R}(s) \sinh \lambda y-\frac{\ell_{3}^{2} \ell_{1}}{\ell_{2}} \gamma A_{\beta} \lambda \sinh \lambda y-w(s+y)+F(s)[\cosh \lambda y+\lambda s \sinh \lambda y] \\
& -\int_{j}^{s+y}\left[\xi F(\xi)+E A u_{R}(\xi)\right] \lambda^{2} \cosh \lambda(s+y-\xi) d \xi
\end{aligned}
$$


This is an integral equation for $F(s)$ given the history $U_{\mathbb{R}}(s)$. If there is no yielding at the pipe stemming interface, $\vec{y}=L-s$, then Eq. (35) can be written

$$
\begin{aligned}
& W+w L=\lambda E A u_{R}(s) \sinh \lambda(L-s)-\frac{l_{3}^{2} l_{1}}{l_{2}} \gamma A_{s} \lambda \sinh \lambda(L-s)+F(s)[\cosh \lambda(L-s)+\lambda s \sinh \lambda(L-3)] \\
& -\int_{1}^{L}\left[\xi F(\xi)+E A u_{R}(\xi)\right] \lambda^{2} \cosh \lambda(L-\xi) d \xi \text {. }
\end{aligned}
$$

To obtain an expression for $F(s)$, Eq. (36) is differentiated with respect to s,

$$
F^{\prime}(3)=\frac{-\frac{l_{3}^{2} l_{1} \gamma}{l_{2}} A_{s} \lambda^{2}-\lambda E A \frac{d u_{R}}{d s} \tanh \lambda(L-s)}{I+\lambda s \tanh \lambda(L-s)} .
$$

This differential equation can be integrated to obtain

$$
F(s)=w+w L+\int_{L}^{s} \frac{\left(\frac{R_{3}^{2} R_{1}}{R_{2}} \gamma A_{s} \lambda^{2}+\lambda E A \frac{d u_{R}}{d \xi} \tanh \lambda(L-\xi)\right) d \xi}{I+\lambda \xi \tanh \lambda(L-\xi)},
$$

where the boundary condition $F(L)=W+$ wL is used. This boundary condition is obtained from Eq. (36) by setting $\mathbf{s}=\mathbf{L}$.

It is more difficult to solve for $F(s)$ when there is some yielding along the pipe-stemming interface. In this case Eq. (35) for F(s) still applies, but in order to solve it one must know $y$, which is a function of $s$, $y=y(s)$. The function $y(s)$ is determined by solving for the depth $s+y$ at which yielding first occurs as a function of s. At yield, $\sigma(x, s)$ and $\tau(x, s)$ are given by Eq. (16); from Eqs. (16) and (5) we ob:ain the condition

$$
2 \mu_{p} K r_{0}=\frac{G}{R_{h}-R_{p}}[u(y+s ; s)-u(y+s, y+s)],
$$

which, using Eq. (17) can be rewritten as

$$
E A[u(s+y, s)-u(s+y, s+y)]=\frac{2 A_{3} l_{3}^{2} \gamma l_{1}}{l_{2}-l_{1}}
$$

When $u(s+y, s)$ from Eq. (31) and $u(s+y, s+y)$ from Eq. (10) are substituted into Eq. (40), an implicit equation for $y(s)$ is obtained:

$\mathrm{EAu}_{R}(\mathrm{~s}) \cosh \lambda y+\frac{\ell_{3}^{2} l_{1} \gamma A_{s}}{\ell_{2}}\left(\frac{l_{1}+\ell_{2}}{\ell_{1}-l_{2}}-\cosh \lambda y\right)+F(s)\left[\frac{1}{\lambda} \sinh \lambda y+s \cosh \lambda y\right]$

$$
-(y+s) F(y+s)-E A u_{R}(y+s)-\int_{s}^{s+y}\left[\xi F(\xi)+E A u_{R}(\xi)\right] \lambda \sinh \lambda(y+s-\xi) d \xi=0 .
$$


The analytical development of the solution stops here. Equations (41) and (35) must be solved simultaneously for the function $y(s)$, which gives the distarice from the free surface of the stemming to the point where the stemming begins to slip relative to the pipe, and the function $F(s)$, which is the force necessary to support the pipe. Once $F(s)$ and $y(s)$ have been determined, the function $T(x, s)$ represerting the axial force along the pipe is completely determined by Eq. (34).

\section{GUIDE FOR PROGRAMMING}

In this section a step-by-step guide is outlined for a computer program for determining the pipe stemming load. The first draft of the computer program should omit Steps 7 and 8 , because that is where the axial force along the length of the entire pipe is calculated. In Steps 3 and 6 the axial force in the pipe at the earth's surface is calculated, and since this force can be comipared with the field data, it is the principal force of interest in the first stage of program development.

Step 1: Introduce the variables.

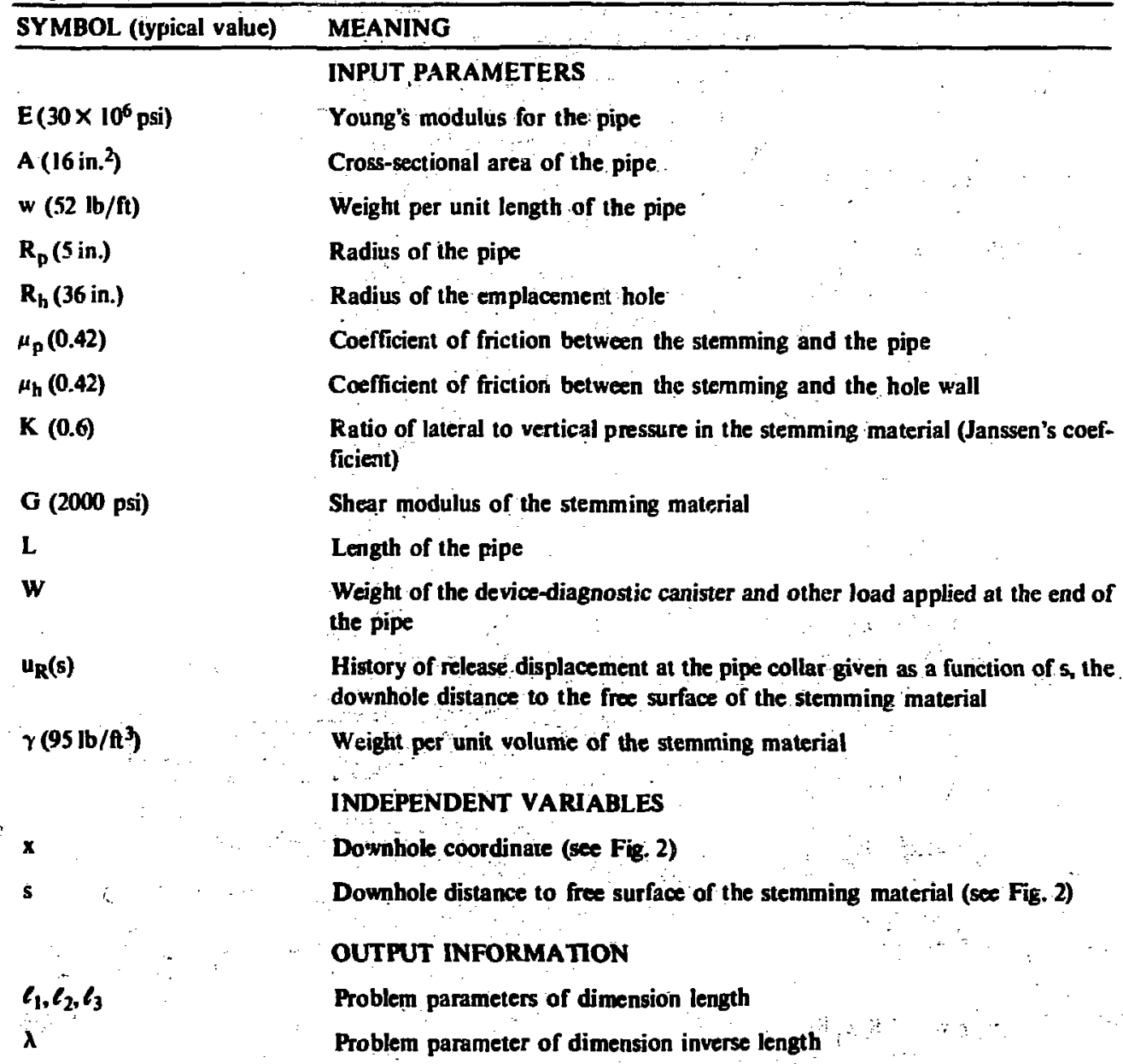


A.

$w_{e}$

$\sigma_{0}$

y(s)

$\mathbf{F}(\mathbf{s})$

$\mathbf{s}_{\mathbf{G}}$

$F_{0}(\mathbf{s})$

$\mathbf{T}(\mathbf{x}, \mathbf{s})$

$T_{0}(x, s)$
Area of annular region between pipe and hole wall

Reduced effective weight per unit length of the pipe

Stress in the stemming material

Distance from the free surface of the stemming material to the point where the stemming material begins to slip relative to the pipe (see Fig. 2)

Force necessary to support the pipe at the earth's surface when there is no slipping between the stemming material and the pipe

Level of the stemming surface when slipping between the stenming material and the pipe begins at the bottom of the pipe, $x=L$

Force necessary to support the pipe at the earth's surface when there is slipping between the stemming material and the pipe

Axial force in the pipe as a function of position along the pipe $x$, and as a function of the distance to the free surface of the stemming $s$, when there is no slipping between the steinming material and the pipe; that is to say $s_{b}$ is not in the interval 0 to $L$, or if it is, then $0<s_{0} \leqslant s<L$.

Axial force in the pipe as a function of position along the pipe $x$, and as a function of the distance to the free surface of the stemming $s$, when there is slipping between the stemming material and the pipe, $0<\mathrm{s}<\mathrm{s}_{\mathrm{o}}<\mathrm{L}$.

\section{Step 2: Preliminary calculations.}

Step du: Using the input data, compute the value of the following quintities

$$
\begin{aligned}
& \ell_{1}=\frac{R_{h}^{2}-R_{p}^{2}}{2 \mu_{h} R_{h} K} \\
& l_{2}=\frac{R_{h}^{2}-R_{p}^{2}}{2 \mu_{p} R_{p}^{K}} \\
& R_{3}=+\left(\frac{E A\left(R_{t}-R_{p}\right)}{2 \pi R_{p} G}\right)^{i / 2} \\
& \lambda=\left(\frac{l_{2}}{\left(l_{1}+l_{2}\right) R_{3}^{2}}\right)^{1 / 2} \\
& A_{1}=\pi\left(R_{h}^{2}-R_{p}^{2}\right) \\
& \sigma_{0}=\frac{\gamma l_{1} l_{2}}{l_{2}-l_{1}} \\
& w_{e}=z-2 \pi R_{p} \mu_{p} K_{0}
\end{aligned}
$$


Step 20: Compute du $u_{R} / d s$ from $u_{R}(s)$. This calculation causes concern because it is suspected that the function $\mathrm{u}_{\mathrm{R}}(\mathrm{s})$ should contain sharp changes from one value to another separated by long periods where it is constant. It is feared that the shape changes in $u_{R}(s)$ will make $d u_{R} / d s$ too high (perkaps infinite), and these shape changes in $u_{R}(s)$ should be smoothed in a reasonable fashion so that the derivative stays bounded. For example if $u_{R}(s)$ has the form

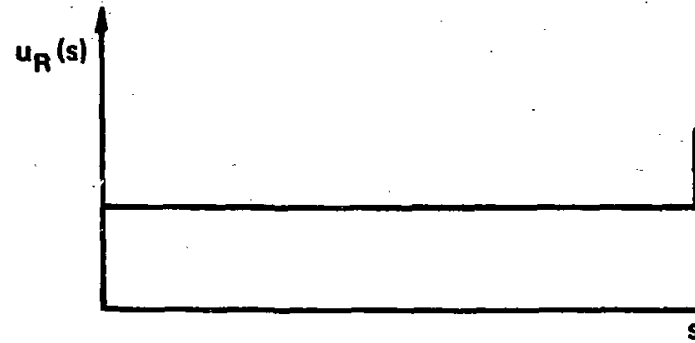

then $\frac{d u_{R}(s)}{d s}$ has the form

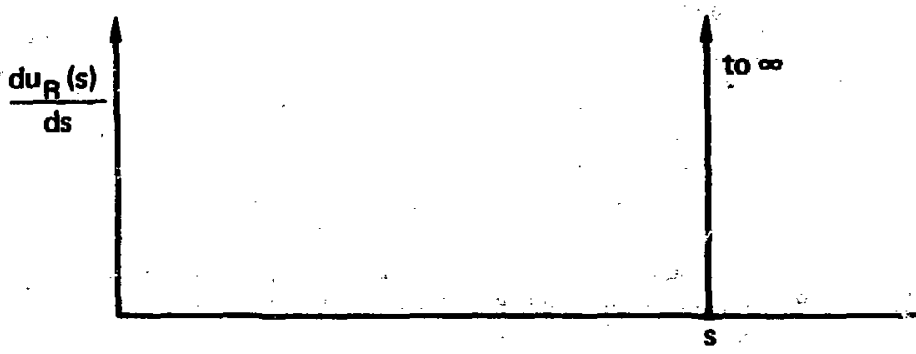

But if we smooth u $(s)$, spreading the shape change, to

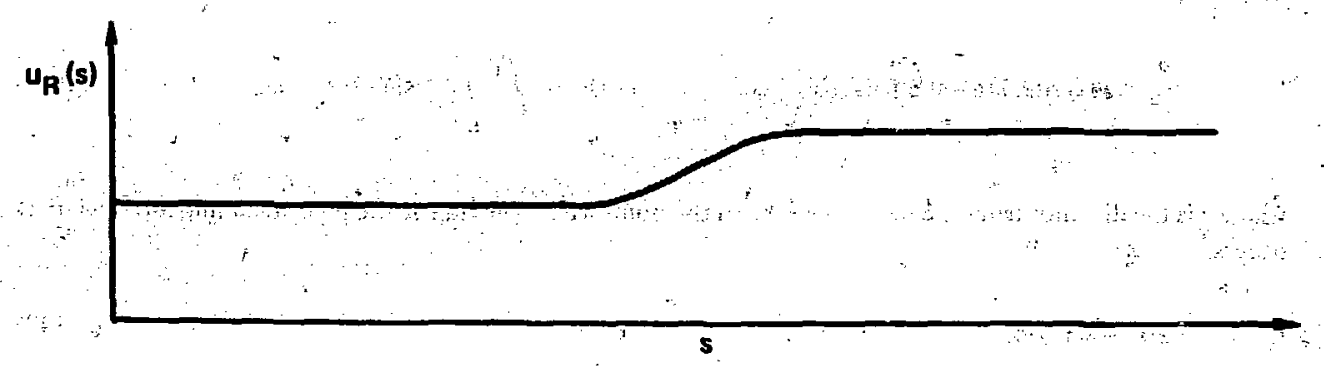


then $\frac{d u_{R}(0)}{d s}$ hes the form

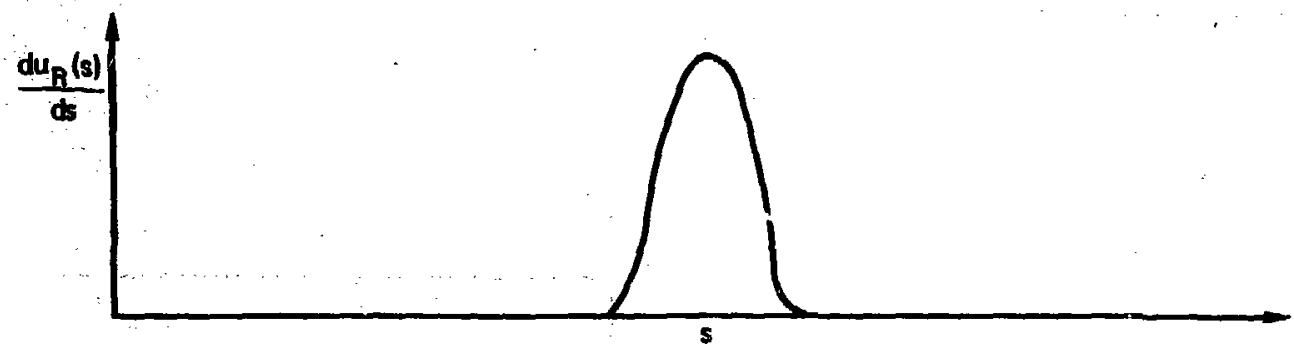

Step 3: Determinetion of the function $F(s)$.

The function $F(s)$ is given by Eq. (38), which is written in the form

$$
F(s)=W+w L+I(s),
$$

where

$$
I(s)=\int_{L}^{3} \frac{\left(\frac{l_{3}^{2} l_{1}}{l_{2}} \gamma A_{s} \lambda^{2}+\lambda E A \frac{d u_{R}}{d \xi} \tanh \lambda(L-\xi)\right) d \xi}{1+\lambda \xi \tanh \lambda(L-\xi)}
$$

This iintegral con be evaluated by any computational method for integrals (e.g. Simpsun's rule). The evaluation should comimence at $s=L$ and procesd downward in the absolute value of $s$ to $s=0$. The funetion $F(s)$ should then be stored.

Prelude to Steps 4 and 5. When there is yielding at the stemming-pipe interface the system of integral Eqs (35) and (41) must be solved for the functions $y(S)$ and $F(s)$ before the axial force $T(4, s)$ in the pipe can be determined. Equations (35) and (41) can be written in the simpler forms

$$
C_{1}+C_{2} z=F(s) \cosh \lambda(z-s)+\left[Q(s)-C_{3}\right] \sinh \lambda(z-s)-\int_{3}^{z} Q(\xi) \lambda \cosh \lambda(z-\xi) d \xi
$$

and

$$
C_{4}=F(s) \sinh \lambda(z-8)+\left[Q(s)-C_{3}\right] \cosh \lambda(z-s)-Q(z)-\int_{s}^{z} Q(\xi) \lambda \sinh \lambda(z-g) d \xi
$$

where 2 is the distance from the top of the pipe to the point where yielding at the pipe stemining interface first oceurs,

$$
z(s)=8 \mp y(s)
$$


$Q(\xi)$ is a notation for a combination of functions, including $\mathbf{F}(\boldsymbol{\xi})$,

$$
Q(\xi) \equiv \lambda\left[\xi F(\xi)+E A u_{R}(\xi)\right]
$$

and $C_{1}, C_{2}, C_{3}$, and $C_{4}$ are constants,

$$
C_{1}=W+w_{e} L, C_{2}=w-w_{e}, C_{3}=\frac{R_{3}^{2} l_{1}}{l_{2}} \gamma A_{3} \lambda, C_{4}=\frac{\gamma A_{1} l_{1}}{\lambda\left(l_{2}-l_{1}\right)} .
$$

Equation (45) is for the determination of $F(s)$ and Eq. (46) is for $2(s)$. These equations must be solved simultaneously. The next step outlined below. solves for the level of free surface of the stemming when yielding begins between the pipe and the stemming material at the bottom of the pipe. This level of the free surface is denoted by $\mathrm{s}_{\mathrm{o}}$. It will be determined from $\mathrm{Eq}$. (46). When $\mathrm{s}$ is in the domain $0<\mathrm{s}_{\mathrm{o}}<\mathrm{s} \leqslant \mathrm{L}$ there is no yielding bet ween the pipe and the stemming, $z(s)$ is either undefined or it can be assigned the value $L$, ano $F(s)$ is given by $\mathrm{Eq}$. (38) or (43). However, when $\mathrm{s}$ is in the range $0<\mathrm{s}<\mathrm{s}_{0}<\mathrm{L}$ there is yielding and Eqs. (45) and (46) must be solved simultaneously for $F(s)$ and $z(s)$. A suggested method for accomplishing this complicated calculation is given in Step 5 below. The curves $F(s)$ and $z(s)$ in the domain $0<s<L$ are given in Fig. 5.

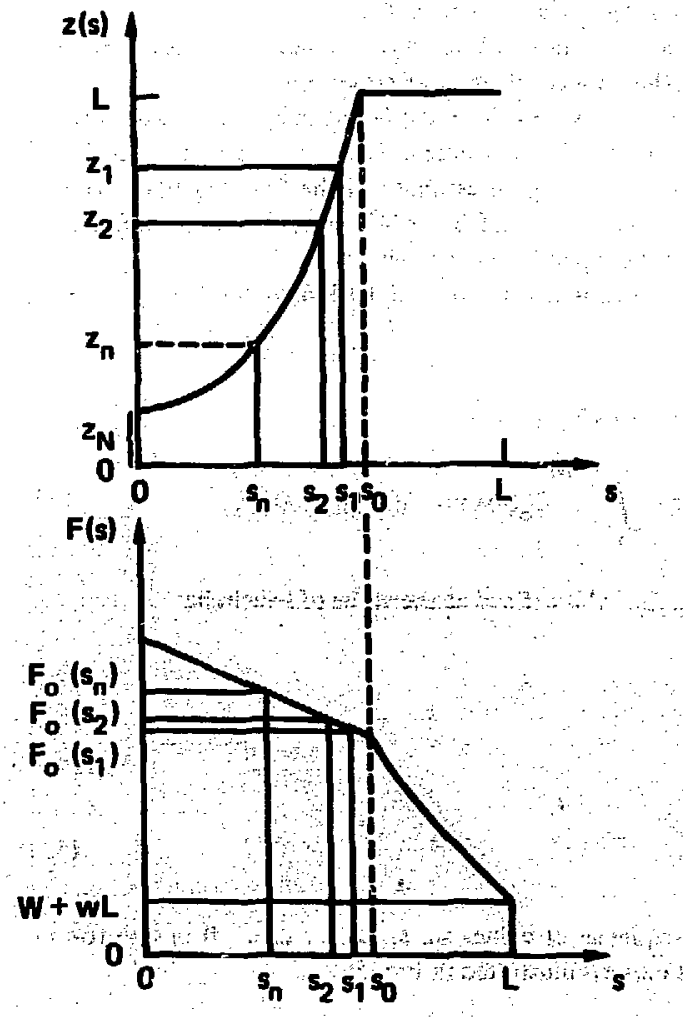

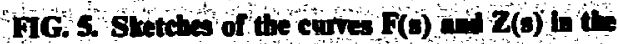




\section{Step 4: Detinination of the level of the steming surace $s_{0}$ whas Ilpphe betreen the stemuing material and the plpe begins at the ciotton of the pipe, $x=L$.}

To find $s_{0}$ we must solve Eq. (46). for $s$ when $z=L$ and $F(s)$ is given by Eq. (38) or (43). Thus we define the function

$$
M_{0}(s)=F(s) \sinh \lambda(L-s)+\left[Q(s)-C_{3}\right] \cosh \lambda(L-s)-Q(L)-\int_{s}^{L} Q(\xi) \lambda \sinh \lambda(L-\xi) d \xi,
$$

and note that $M_{0}(L)=-C_{3}$. Equation (46) is satisfied identically when $M_{0}(s)=C_{4}$, thus $\delta_{0}$ is defined as the value of $s$ such that

when

$$
\mathbf{M}_{0}(\mathbf{s})=\mathbf{C}_{4}
$$

then

$$
s=s_{0} .
$$

The calculations of $M_{0}(s)$ should start at $s=L$ when $M_{0}(L)=-C_{3}$ and proceed in increments until $M_{0}(s)=C_{4}$, at which point $s_{0}$ will be determined.

Step 5: Determination of the functions $F(s)$ and $y(s)$ in the domain $0<s \leqslant 8<L$.

The method suggested hare for the simultaneous solution of Eqs. (45) and (46) may be primitive; it is possible that the software exists for the solution of this system of integral equations.

The method suggested is as follows. Starting at $s=s_{0}, z=L$, and $F(s)$ given by Eq. (38) or (43), the system of Eqs. (45) and (46) will be solved in step increments of magnitude $q$ from $s=s_{0}$ to $s=0$. At the $n^{\text {th }}$ increment we set $z=z_{n}=L-q n$ and silve Eq. (46) for $s_{n}$ using an estimate of the function $F(s)$ given by Eq. (45) in the provious step. The first estimate of $F(s)$ is given by Eq. (38) or (43) and it has been computed in Step 3. The notation $F_{0}(s)$ is used to denote the force at the top of the pipe when $s<s_{0}$.

To find $s_{n}$ we must solve Eq. (46) for $s$ when $z=z_{n}=L-q$ and $F_{0}(s)$ is known for $0<s_{n}<s<L$. Thus we define the function

$$
\begin{aligned}
M_{n}(s)=F_{0}(s) \sinh \lambda(L-n q-s)+ & {\left[Q_{0}(s)-C_{3}\right] \cosh \lambda(L-n q-s) } \\
& -Q_{0}(L-n q)-\int_{0}^{L-n q} Q_{0}(\xi) \lambda \sinh \lambda(L-n q-\xi) d \xi .
\end{aligned}
$$

Equation (46) is satisfied identically when $M_{n}(s)=C_{4}$, thus $s_{n}$ is defined as the value of $s$ such that

when

$$
M(s)=C_{4}
$$

then

$$
\mathbf{s}=\mathbf{n}
$$

The curve $z=z(s)$ can then be determined as the sequence of values $s_{0}, s_{1}, s_{2}, \ldots, 0$ in one-to-one correspondence with the values $L, z_{1}, z_{2}, \ldots, z_{n}$ This curve is illustrated in Fig. 5 . 
At each step in the calculation above the value of $F_{0}(s)$ at $s_{n}$ must be determined. The value of $F(s)$ for $0<s_{0}<s<L$ is given by Eq. (38) or (43), thus $F\left(s_{0}\right)$ is known. To determine $F_{0}\left(s_{n}\right)$ Eqs. (46) and (48) are solved for $F_{0}(s)$ and we write

$$
\begin{array}{r}
{\left[\cosh \lambda\left(z_{n}-s_{n}\right)+\lambda s_{n} \sinh \lambda\left(z_{n}-s_{n}\right)\right] F_{a}\left(s_{n}\right)=C_{1}+C_{2} z_{n}+\left[C_{3}-\lambda E A u_{R}\left(s_{n}\right)\right] \sinh \lambda\left(z_{n}-s_{n}\right),} \\
-\int_{s_{n}}^{z_{n}} Q_{0}(\xi) \lambda \cosh \lambda\left(z_{n}-\xi\right) d \xi,
\end{array}
$$

where $z_{n}=L-q n$. The integral on the right will be based on the values of the $F_{0}(s)$ through $s_{n}-1$, with some estimate made for the value $s_{n}$. This calculation should be iterated with the solution for $s_{n}$ to obtain both $F_{o}\left(s_{n}\right)$ and $s_{n}$ to the desired accuracy. The curve $F_{0}(s)$ is illustrated in Fig. 5. It should be kept in mind that the portion of the curve in the domain $s_{0}>s>L, F(s)$, is given by Eq. (38) or (43) and only that portion of the curve in the domain $0<s<s_{0}, F_{0}(s)$ must be calculated by this iterative scheme.

This iterative scheme proceeds until the value of $s_{n}<0$ occurs. The calculation is then terminated. The number of steps to achieve $s_{n}<0$ is denoted by $N$, thus $z_{N}$ is the value of $z$ corresponding to $s=0$.

Before proceeding to the next step compute $y(s)=z(s)-s$.

Prelade to Steps 7 and 8 . It should be kept in mind that $T(x, s)$ represents the axial force in the pipe when there is no slipping at the pipe stemming interface: This situation pertains if $s_{0}$ does not exist in the interval 0 to $L$ or if $0<s_{0} \& s<L$. $T_{j}(x, s)$ represents the axial force in the pipe when there is slipping. $0<s<s_{0}<L$. Thus, except for the single situation when both $T(x, s)$ and $T_{0}(x, s)$ give the same answer; that is when $s=s_{0}$, the two calculations for the axial force, that is $T(x, s)$ and $T_{0}(x, s)$, are mutually exclusive. For any particular value of s either Step 7 or 8 applies, but not both.

\section{Step 7: Determination of $T(x, s)$.}

This is, of course, a more laborious calculation because $T(x, s)$ is a function of two variables. It is suggested that this calculation be done by/ lixing on a value of $s$ and then detemining the function of $x$, then incrementing in $s$ and determining the new function of $x$, etc. One should start at $s=L$, of course. $T(x, s)$ is given b:

$$
T(x, s)=\dot{F}(s)-w x \text { for } 0 \leqslant x \leqslant s
$$

and

$$
\begin{array}{r}
T(x, s)=\lambda E A u_{R}(s) \sinh \lambda(x-s)-\frac{l_{3}^{2} l_{1}}{l_{2}} \lambda A_{s} \gamma \sinh \lambda(x-s)-w x+F(s)[\cosh \lambda(x-s)+\lambda s \sinh \lambda(x-s)] \\
\int_{s}^{x}\left[\xi F(\xi)+E A u_{R}(\xi)\right] \lambda^{2} \cosh \lambda(x-\xi) d \xi \text { for } s \leqslant x<L
\end{array}
$$

These results follow from Eqs. (8) and (34).

\section{Step 8 s Deteriminatio of $T_{d}(x ; 0)$.}

This is an even more laborious calculation because $T_{0}(x, s)$ is a function of two variables and is specified by a different expression in three domains. $T_{0}(x, s)$ is given by

$$
T_{0}(x, s)=F_{0}(s)-w x \text { for } 0<x<s \text {, }
$$




$$
T_{0}(x, s)=\lambda E A u_{R}(s) \sinh \lambda(x-s)-\frac{l_{3}^{2} l_{1}}{l_{2}} \gamma A_{s} \lambda \operatorname{sirh} \lambda(x-s)-w x+F_{0}(s)[\cosh \lambda(x-s)+\lambda s \sinh \lambda(x-s)]
$$

for $s \leqslant x \leqslant s+y$

$$
T_{0}(x, s)=W+w_{e}(L-x) \text { for } s+y<x \leqslant L
$$

These results follow from Eqs. (8), (22) and (34).

\section{ACKNOWLEDGMENTS}

I would like to acknowledge valuable discussions with Alex Blake, Will Burden, Hank Knoll, Dennis Maiden, Frank Morrison, Jim Quigg, Larry Starrh, Landruff Trent, Hugh Watling and Max Zaslawsky at Livermore and with Ed Conway of the Department of Mathematics at Tulane University. In particular, it was Frank Morrison's idea to use the zero-strain reference state. The method of solution of ihe governing system of differential equations was suggested by Ed Conway. Both Frank Morton and Jim Quigg have checked portions of my calculations and I appreciate their efforts.

\section{REFERENCES}

1. W. A. Hamilton, Stemming Loads on Emplacement Syste.s, Lawrence Livermore Laboratory, Livermore, CA, ANS 9-3288 (1969).

2. J. L. Cramer, Stemming Test Repon, Lawrence Livermore Laboratory, Livermore, CA, L6-5110 (1965).

3. D. Norris, Stemming Loads-A Mathematical Model, Lawrence Livermore Laboratory, Livermore, CA, ENN $70-48$ (1970):

4. D. Norris, J. Staats, and W.Kleck, stem-A Computer Program for Calculation on Stemming Loads, Lawrence Livermore Laboratory, Livermore, CA, UCID-30049 (1972).

5. A. Blake 1 Brief Review of Stemming Loads, Theory and Experiment, Lawrence Livermore Laboratory, Livermore, CA, unpubilished report (August 29, 1973).

6. S. C. Cowin, "The Theory of Static Loads in 'Bins," J. Appl. Mech., 44, 409 (1977).

7. V. Sundaram and S. C. Cowin, “A Reassessment of Static Bin Pressure Experiments," Powder Tech. 22, 23 (1979):

8. S.C. Cowin, "The Pressure Ratio in the Theory of Bin Pressures," J. Appl Mech. 46, 524 (1979).

9. T. M. Apostol, Mathematical Analysts, Addison Wesley Pübl. Co., Reading, Mis (1957), p. 220. 


\section{APPENDIX I-THE SOLUTION WITHOUT THE APPROXIMATION}

The approximation made in Sec. 4 simplified the solution, but it was not necessary to make the approximation to obtain the solution. Here the solution to the problem with no approximations is sketched. Appendix I-I and Appendix I-2 parallel Secs. 5 and 6. The results of this Appendix support the assumed approximation because they show that the effects of the neglected term in Eq. (3) attenuate seven times more rapidly than the effects of the terms retained. It is also shown that there is no more formal difficulty in the solution of the equations without the approximation, although the algebra is more awkward.

\section{I-I THE SOLUTION IN THE LOWER PORTION \\ OF THE STEMMED REGION WHEN SLIPPING OCCURS ON THE PIPE-STEMMING INTERFACE}

$\therefore \quad$ onsider the problem in two parts. Here we consider the case when $\tau=-\mu_{\mathrm{p}} \mathrm{K} \sigma$ in the lower portion of $t h c$ semmed region. Then, in Sec. 1-2, we consider the case when $\tau(x, s)=-\mu_{p} K \sigma$ in the lower portion of the sic inmed region and $r(x, s)>-\mu_{p} K \sigma(x, s)$ in the upper portion of the stemmed region. That is, we consider hc tho simpler problein of no slipping along the lower portion of the pipe stemming interface, and in the nex section we use this result to obtain a solution to the problem in the no-slipping region above the slipping portior.

Let $s+y, y>0$ denote the downhole distance at which slip first occurs, $0<s+y<x<L$. Slip is assumed to occur along, the pipe for $x$ greater than $s+y$ and less than $L, s+y<x<L$. For these values of $x$, $\tau(x, s)=-\mu_{p} K \sigma(x, s)$ and Eqs. (2b) and (3) take the form

$$
\frac{d T(x, s)}{d x}-\alpha \sigma+w=0 \text { for } s+y \leqslant x \leqslant L
$$

and

$$
\frac{\mathrm{da}(\mathrm{x}, \mathrm{s})}{\mathrm{dx}}+\frac{N}{l_{1}} \sigma(x, s)-\gamma=0 \text { for } s+y \leqslant x \leqslant L
$$

where

$$
\alpha=2 \pi K \mu_{p} R_{p}
$$

and

$$
\mathbf{N} \equiv 1-\frac{\mathbf{R}_{\mathrm{p}} \boldsymbol{\mu}_{\mathrm{p}}}{\mathbf{R}_{\mathrm{h}} \boldsymbol{\mu}_{\mathrm{h}}}
$$

The quantity $\mathbf{N}$ is equal to î then there is no pipe in the hole. In general $0<N<1$. The boundary conditions used for the solution of Eqs. (I-1) and (1-2) are

$$
\underbrace{\begin{array}{c}
\sigma(x, s)|=\sigma(s+y, s), T(x, s)|=W \\
x=s+y
\end{array}}_{1} \underbrace{x=L}_{2}
$$


where $W$ is the weight of the canister. The solution to the differential equation (I-2) using the boundary condition $\mathbf{E q} .(\mathrm{I}-\mathbf{j})_{1}$ is

$$
a(x, s)=\frac{\gamma \ell_{1}}{N}+\left[o(s+y, s)-\frac{r \ell_{1}}{N}\right] e^{-(x-s-y)\left(N / R_{1}\right)}, s+y \leqslant x<L .
$$

The solution to the differential equation (l-1) subject to the boundary condition Eq (I-5) 2 is

$$
T(x, s)=W+\left(w-\frac{\sigma \gamma R_{1}}{N}\right)(L-x)-\frac{\alpha l_{1}}{N}\left[\sigma(y, s)-\frac{\gamma \ell_{1}}{N}\right]\left[\mathrm{e}^{-(x-s-y)\left(N / \ell_{1}\right)}-e^{-(L-s-y)\left(N / l_{1}\right)}\right]
$$

In the special case when the unslipped zone is very short, we may introduce the approximation $\mathrm{y} \cong 0$ and observe that

$$
\sigma(s, s)=0
$$

then Eq. (1-6) becomes

$$
\sigma(x, s)=\frac{r l_{1}}{N}\left(1-\mathrm{e}^{-\left(\mathrm{N} / \mathrm{g}_{1}\right)(\mathrm{x}-\mathrm{s})}\right), \mathrm{s} \leqslant \mathrm{x} \leqslant \mathrm{L}
$$

and the tension in the pipe is given by

$$
T(x, s)=W+\left(w-\frac{\alpha y l}{N}\right)(L-x)+\frac{\alpha l_{1}^{2}}{N^{2}}\left[e^{-(x-s)\left(N / R_{1}\right)} e^{-(L-s)\left(N / l_{1}\right)}\right] \text { for } s \leqslant x \leqslant L .
$$

The tension in the pipe at the point $x=s$ for $y \cong 0$ is, from Eq. (I-10), given by

$$
T(s, s)=W+\left(w-\frac{\alpha \gamma l}{N}\right)(L-s)+\frac{\alpha \gamma l_{1}^{2}}{\mathrm{ls}^{2}}\left[1-\mathrm{e}^{-(\mathrm{L}-s)\left(\mathrm{N} / \mathrm{l}_{1}\right)}\right] ;
$$

thus from Eq. (8) the tension at the collar is

$$
F(s)=W+w L-\frac{\alpha \gamma l_{1}}{N}(L-s)+\frac{\alpha r l_{1}^{2}}{N^{2}}\left[1-e^{-(L-s)\left(N / l_{1}\right)}\right]
$$

and the tension in the region $0<x<s$ is

$$
T(x, s)=W+w(L-x)-\frac{\alpha \gamma l_{1}}{N}(L-s)+\frac{\alpha l_{1}^{2}}{N^{2}}\left[1-e^{-(L-s)\left(N / l_{1}\right)}\right]
$$

If there is no stemming in the hole $(\gamma=0$ or $s=L)$, then $T(x, L)$ is given by

$$
T(x, L)=W+w(L-x), \text { for } 0 \leqslant x \leqslant L .
$$


Comparison of Eqs. (I-13) and (I-14) shows

$$
T(x, s)=T(x, L)-\frac{\alpha \gamma l_{1}}{N}\left\{L-s+\frac{l_{1}}{N}\left[1-e^{-(L-s)\left(N / R_{1}\right)}\right]\right\}
$$

and since the second term is always nonpositive, it follows that $T(x, s)$ is less than $T(x, L)$. Thus, slipping along the entire length of the pipe-stemming interface reduces the pipe tension. This occurs because the friction force has been completely reversed and, instead of the pipe supporting stemming material by friction, the pipe is now being partially supported by the stemming material through friction.

\section{I-2 THE SOLUTION IN THE UPPER PORTION OF THE STEMMED REGION WHERE SLIP DOES NOT OCCUR ON THE PIPE-STEMIMING INTERF ACE}

In this case the system of differential equations (1), (2b), (3) and (5) can be reduced to a single thirdorder linear differential equation with constant coefficients for $u(x, s)$. To obtain this, we first substitute Eqs. (1) and (5) into Eq. (2b) and solve for $\alpha(x, s)$

$$
\sigma(x, s)=\frac{E A l_{2}}{A_{s} l_{3}^{2}}[u(x, s)-u(x, x)]-\frac{l_{2}}{A_{s}}\left[E A u^{\prime \prime}(x, s)+w\right]
$$

where the following notations have been introduced

$$
\ell_{2}=\frac{R_{h}^{2}-R_{p}^{2}}{2 \mu_{p} R_{p} k}, \ell_{3}=+\left(\frac{E A\left(R_{h}-R_{p}\right)}{2 \pi R_{p} G}\right)^{1 / 2}, A_{s}=\pi\left(R_{h}^{2}-R_{p}^{2}\right)
$$

The differential equation for $u(x, s)$ is obtained by substituting $\sigma(x, s)$ given by Eg. (I-16) into

$$
\sigma^{\prime}(x, s)+\left(\frac{1}{l_{1}}+\frac{1}{l_{2}}\right) \sigma(x, s)-\frac{E A}{l_{3}^{2} A}[u(x, s)-u(x, x)]-\gamma=0
$$

a result that is obtained by placing Eq. (5) into Eq. (3),

$$
u^{\prime \prime \prime}(x, s)+\left(\frac{1}{l_{1}}+\frac{1}{l_{2}}\right) u^{\prime \prime}(x, s)-\frac{1}{l_{3}^{2}}\left[u^{\prime}(x, s)-u^{\prime}(x, x)\right]-\frac{1}{l_{1} l_{3}^{2}}[u(x, s)-u(x, x)]+\frac{1}{l_{4}^{2}}=0 \text {, }
$$

where

$$
\left.\frac{1}{l_{4}^{2}}=\frac{/ 1}{\left(l_{1}\right.}+\frac{1}{l_{2}}\right) \frac{w}{\mathrm{AE}}+\frac{\gamma \mathrm{A}_{s}}{l_{2} \mathrm{EA}}
$$

We, are interested in the solution of the differential equation (I-19) in the region $s<x<s+y$, i.e., the stemmed region above the region where slipping has occured. The solution of Eq: (1-19) in the case where no slipping occurs in the stemmed region is obtained from this solution by setting $s+y$ equal to $L$.

The functions $u(x, x)$ and $u^{\prime}(x, x)$ are obtained from $u(x, s)$ for the region $0<x<s$, Eq. (10), by evaluating $u(x, s)$ at $x=s$ and $u^{\prime}(x, s)$ at $x=s$ and then setting $x=s$,

$$
u(x, x)=\frac{F(x)}{E A} x-\frac{w}{2 E A} x^{2}+u_{R}(x), \quad u^{\prime}(x, x)=\frac{F(x)}{E A}-\frac{w}{E A} x .
$$


The differential equation governing $u(x, s)$ in the region $s<x \leqslant y$ is obtained by substituting Eq. (1-21) into Eq. (I-19),

$$
\begin{aligned}
u^{\prime \prime \prime}(x, s)+\left(\frac{1}{l_{1}}+\frac{1}{l_{2}}\right) u^{\prime \prime}(x, s) & -\frac{1}{l_{3}^{2}} u^{\prime}(x, s)-\frac{1}{l_{1} l_{3}^{2}} u(x, s) \\
& =-\frac{1}{l_{4}^{2}}-\frac{u_{R}(x)}{l_{1} l_{3}^{2}}+\frac{w x}{E^{2} l_{3}^{2}}\left(1+\frac{x}{2 l_{1}}\right)-\frac{F(x)}{E_{2} l_{3}^{2}}\left(1+\frac{x}{l_{1}}\right)
\end{aligned}
$$

The boundary conditions that apply to Eq. (I-22) at $x=s$ are that $u(x, s), u^{\prime}(x, s)$ and $u^{\prime \prime}(x, s)$ be coritinuous. Since $u(x, s)$ is given by $\mathrm{Eq}$. (10) for $0 \leqslant x \leqslant s$, we can write

$$
\begin{aligned}
& u(s, s)=\frac{s F(s)}{E A}-\frac{w}{2 E A} s^{2}+u_{R}(s) \\
& u^{\prime}(s, s)=\frac{F(s)}{E A}-\frac{w}{E A} s \\
& u^{\prime \prime}(s, s)=-\frac{w}{E A} .
\end{aligned}
$$

$u(x, s)$ is continuous at $x=s$ because the displacement is continuous, and $u^{\prime}(x, s)$ is continuous because the axial force in the bar is continuous [see Eq. (1)]. To see that $u^{\prime \prime}(x, s)$ is continuous, place Eq. (1) in both Eqs. (2a) and (2b). The limit as $x \rightarrow s^{-}$of $u^{\prime \prime}(x, s)$ is given by Eq. (2a) and the limit as $x \rightarrow s^{+}$of $u^{\prime \prime}(x, s)$ is given by Eq. (2b). These two limits coincide since $r(x, x)=0$.

The solution to the homogeneous part of Eq. (I-23) is

$$
u_{H}(x, s)=a_{1} e^{\lambda_{1} x}+a_{2} e^{\lambda_{2} x}+a_{3} e^{\lambda_{3} x}
$$

where $a_{1}, a_{2}$, and $a_{3}$ are constants and $\lambda_{1}, \lambda_{2}$ and $\lambda_{3}$ are the solutions to the cubic equation

$$
\lambda^{3}+\left(\frac{1}{\ell_{1}}+\frac{1}{\ell_{2}}\right) \lambda^{2}-\frac{1}{\ell_{3}^{2}} \lambda-\frac{1}{\ell_{1} \ell_{3}^{2}}=0
$$

It is easy to show that $\lambda_{1}, \lambda_{2}$, and $\lambda_{3}$ are related to $\ell_{1}, \ell_{2}$, and $\ell_{3}$ by

$$
\lambda_{1} \lambda_{2} \lambda_{3}=\frac{1}{\ell_{1} \ell_{3}^{2}}, \lambda_{1}+\lambda_{2}+\lambda_{3}=-\frac{1}{l_{1}}-\frac{1}{\ell_{2}}, \lambda_{1} \lambda_{2}+\lambda_{2} \lambda_{3}+\lambda_{1} \lambda_{3}=-\frac{1}{\ell_{3}^{2}}
$$

It can also be shown that since $\ell_{1}, \ell_{2}$ and $\ell_{3}$ are positive, the three roots $\lambda_{1}, \lambda_{2}$ and $\lambda_{3}$ are all real and in general distinct. Furthermore, two are negative and one is positive. The solution to the homogeneous part of Eq (1-22) subject to the boundary condition $\mathrm{Eq}$ (I-23) is

$u_{H}(x, s)=\left[u_{R}(s)+\frac{1}{E A}\left(s F(s)-\frac{w s}{2}\right)\right] \frac{1}{\ell_{1} l_{3}^{2}} g(x-s)+\frac{1}{E A}\left\{[F(s)-w s]\left(\frac{1}{l_{1}}+\frac{1}{l_{2}}\right)-w\right\}$

$$
X g^{\prime}(x-s)+\frac{1}{E A}[F(s)-w s] g^{\prime \prime}(x-s) \text {, }
$$


where the function $g(x)$ is given by

$$
g(x)=\frac{1}{\Delta}\left(\frac{\lambda_{3}-\lambda_{2}}{\lambda_{1}} \mathrm{e}^{\lambda_{1} \mathrm{x}}+\frac{\lambda_{1}-\lambda_{3}}{\lambda_{2}} \mathrm{e}^{\lambda_{2} \mathrm{x}}+\frac{\lambda_{2}-\lambda_{1}}{\lambda_{3}} \mathrm{e}^{\lambda_{3} \mathrm{x}}\right)
$$

and where

$$
\Delta=\left|\begin{array}{ccc}
1 & 1 & 1 \\
\lambda_{1} & \lambda_{2} & \lambda_{3} \\
\lambda_{1}^{2} & \lambda_{2}^{2} & \lambda_{3}^{2}
\end{array}\right|
$$

It is easy to verify that $g(x)$ satisfies the differential equation

$$
g^{\prime \prime \prime}(x)+\left(\frac{1}{\ell_{1}}+\frac{1}{l_{2}}\right) \ell^{\prime \prime}(x)-\frac{1}{\ell_{3}^{2}} B^{\prime}(x)-\frac{1}{\ell_{1} \ell_{3}^{2}} g(x)=0,
$$

and the boundary conditions

$$
g(0)=\ell_{1} \ell_{3}^{2}, g^{\prime}(0)=0, g^{\prime \prime}(0)=0, g^{\prime \prime \prime}(0)=1, g^{\prime \prime \prime \prime}=-\frac{1}{l_{1}}-\frac{1}{l_{2}}
$$

The function $g(x)$ plays an iryportant role in the solution to this problem and we shall consider here its numerical value. If we take a typical emplacement, assuming

$$
\begin{aligned}
& R_{h}=36 \text { in., } R_{p}=5 \text { in., } A=16 \text { in. }^{2}, k \mu_{p} \cong k \mu_{h} \cong \frac{1}{4}, G=2000 \mathrm{psi}, \\
& E=30,000,000 \mathrm{psi},
\end{aligned}
$$

then from Eqs. (4) and (1-17)

$$
\ell_{1}=5.88 \mathrm{ft}, \ell_{2}=42.36 \mathrm{ft}, l_{3}=40.55 \mathrm{ft} .
$$

When the values of Eq. (I-33) are placed in the cubic equation (I-25), the three roots are found to be

$$
\begin{aligned}
& \lambda_{1}=-0.19439 \mathrm{ft}^{-1}=\frac{1}{5.14 \mathrm{ft}}, \\
& \lambda_{2}=-0.02287 \mathrm{ft}^{-1}=\frac{1}{43.72 \mathrm{ft}}, \text { and } \\
& \lambda_{3}=+0.02326 \mathrm{ft}^{-1}=\frac{1}{43 \mathrm{ft}}
\end{aligned}
$$

and from Eq. (I-29), $\Delta$ is given by

$$
\Delta=0.001722 \mathrm{ft}^{-3}=\frac{1}{580.69 \mathrm{ft}^{3}}
$$


When the numerical values of Eqs. (1-34) and (1-35) are substituted into Eq. (1-28), we find

$$
g(x)=\left(-137.8 e^{-x / 5.14}+5526.6 e^{-x / 43.72}+4282.1 e^{+x / 43}\right) \mathrm{ft}^{-3}
$$

where $\mathrm{x}$ is in feet.

With these preliminary results, the formal solution to the differential equation (I-22) can be written, subject to the boundary conditions Eq. (I-23)

$$
u(x, s)=u_{H}(x, s)+\frac{l_{1} \ell_{3}^{2}}{l_{2} E A} A_{s} \gamma-\frac{w x^{2}}{2 E A}-\frac{1}{E A l_{3}^{2}} \int_{s}^{x} g^{\prime}(x-\xi)\left[\frac{E A u_{R}(\xi)}{l_{1}}+F(\xi)\left(1+\frac{\xi}{l_{1}}\right)\right] d \xi
$$

where $g^{\prime}(x-\xi)$ is the Green's function and is the derivative of the function defined by Eq. (I-28), and $u_{H}(x, s)$ is given by Eq. (I-27). The solution (1-37) is a formal solution because the load at the collar $F(s)$ is not known. $F(s)$ will be determined below by matching the boundary conditions at $x=s+y$, that is to say at the interface between the region where slipping occurs and the region where slip does not occur. The boundary conditions are that $T(x, s)$ and $\sigma(x ; s)$ are continuous at $x=s+y . T(x, s)$ in the region $s<x<s+y$ is obtained by substituting Eq. (I-37) into Eq. (1)

$$
T(x, s)=E A u_{H}^{\prime}(x, s)-w x-\frac{1}{\ell_{3}^{2}} \int_{s}^{x} g^{\prime}(x-\xi)\left[\frac{E A}{\ell_{t}} u_{R}(\xi)+F(\xi)\left(1+\frac{\xi}{\ell_{1}}\right)\right] d \xi,
$$

where the fact that $g^{\prime}(0)=0$ has been used. $\sigma(x, s)$ in the region $s<x<s+y$ is obtained by substituting Eq. (1-37) into Eq. (I-16)

$$
\begin{aligned}
& \sigma(x, s)=R_{1} \gamma+\frac{E A l_{2}}{l_{3}^{2} A_{s}}\left[u_{H}{ }^{\prime \prime}(x, s)-l_{3}^{2} u_{H}(x, s)\right]-\frac{l_{2}}{A_{j} l_{3}^{4}}\left\{x F(x)+u_{R}(x)\right. \\
&\left.+\int_{s}^{x}\left[g^{\prime}(x-\xi)-l_{3}^{2} g^{\prime \prime \prime}(x-\xi)\right]\left[u_{R}(\xi)+F(\xi)\left(1+\frac{\xi}{l_{1}}\right)\right] d \xi\right\} .
\end{aligned}
$$

This calculation is not recorded beyond this point. Formally, the calculation is identical with the one performed in Sec. 6 for the approximate case. The difficulty is the awkward and tedious algebra. 


\section{APPENDIX II-THE EFFECT OF TEMPERATURE ON THE DETERMINATION OF THE PIPE-STEMMING LOAD}

Temperature distribution has a considerable effect on the support pipe tension. In the analysis of the effect of temperature on pipe tension, it can be assumed that the mechanical problem does not influence the thermal problem, although the converse is untrue. Thus, the determination of the temperature distribution along the pipe from the given or assumed thermal states of the pipe and stemming material can be solved independent of the mechanical problem of determining the support pipe tension. It is assumed that this has been done and the temperature $\theta$ is $\mathrm{known}$ as a function of downhole distance $x$ and time $t, \theta=\theta(x, t)$. The temperature distribution $\theta=\theta\left(x, t_{R}\right)$ at some particular time is used as reference. The temperature distribution in the pipe will cause the pipe to change length. Let $u_{T}(x, t)$ denote the displacement at $x$ due to the temperature change from time $t_{R}$, then

$$
\frac{d u_{T}}{d x}=\frac{\alpha}{1-2 v}\left[\theta(x, t)-\theta\left(x, t_{R}\right)\right]
$$

or

$$
u_{T}=\frac{\alpha}{1-2 \nu} \int_{0}^{x}\left[\theta(\xi, t)-\theta\left(\xi, t_{R}\right)\right] d \xi
$$

where $\alpha$ is the coefficient of linear thermal expansion and $v$ is the Poisson's ratio of the pipe.

A complication of the mechanical problem caused by thermal effects is that time $t$ now becomes an independent variable in the mechanical problem. Thus the axial force $T$ depends upon $x, 5$ and $t$ as does the displacement $u$, the shear stress $r$ and the vertical stress $\sigma$ in the stemming material. If $s$ is known as a function of time, then $\mathrm{T}$ is again a function of two independent parameters. Assume that $\mathrm{s}=\mathrm{s}(\mathrm{t})$ is known. Then we can write $T(x, t)$ for $T(x, s(t), t)$ and similarly for the other independent variables. With this notation Eq. (1) is replaced by

$$
T(x, t)=E A\left\{\frac{\partial u(x, t)}{\partial x}-\frac{a}{1-2 \nu}\left[\theta(x, t)-\theta\left(x, t_{R}\right)\right]\right\} .
$$

The equations governing the equilibrium of the pipe and stemming. Eqs. (2a), (2b) and (3), are unchanged except for independent variables

$$
\begin{aligned}
& \frac{d T(x, t)}{d x}+w=0, \quad \text { if } 0<x<s(t), \\
& \frac{d T(x, t)}{d x}+2 \pi R_{p} \tau(x, t)+w=0, \quad \text { if } s(t)<x<L, \\
& \frac{d \sigma(x, t)}{d x}+\frac{\sigma(x, t)}{\ell_{i}}+\frac{2 R_{p}}{R_{h}^{2}-R_{p}^{2}} \quad T(x, t)-\gamma=0, \text { if } s(t)<x<L,
\end{aligned}
$$

and

$$
\sigma(x, t)=0 \text { if } 0<x<s(t)
$$


The constitutive equation specifying the shear stress at the pipe-stemming interface must be modified to account for the length changes caused by the temperature distribution. In the present situation.

$$
f(x, t)=\mu_{p} K \sigma(x, t)-\frac{G}{R_{n}-R_{p}}\left\{u(x, t)+\frac{\alpha}{1-2 v} \int_{0}^{x}\left[\theta(\xi, t)-\theta\left(\xi, t_{R}\right)\right] d \xi-u\left(x, t^{*}\right)\right\}
$$

where $t^{*}$ is the time when $s$ passed the station $x, t^{*}=s^{-1}(x)$, which can be obtained from the known function s(t) by inversion.

Since $\theta(x, t)$ and $s(t)$ are assumed to be known, the solution of the combined thermal and mechanical problem to determine the collar load of the support pipe is not, in principle, any more difficult than the solution of the mechanical problem alone. The algebraic manipulations may be more complicated, but that will be a minor matter. 